\title{
Anomalous Cognition in the Context of Time: Does the Viewer Describe a Deterministic or a Probabilistic Future?
}

\author{
MaXimilian Müller \\ Helmut Schmidt University/University of the Federal Armed Forces, Hamburg, Germany \\ maxm@gmx.eu
}

\section{Marc WitTMANN}

Institute for Frontier Areas of Psychology and Mental Health, Freiburg, Germany wittmann@igpp.de

Submitted August 3, 2020; Accepted April 16, 2021; Published September 30, 2021

https://doi.org/10.31275/20211923

Creative Commons License CC-BY-NC

\begin{abstract}
In this process-oriented study, we examined the influence of the time dimension on Psi effects in two experimental conditions (present vs. future). For data collection, selected viewers with experience in the remote viewing method gathered information about targets that were distant in space (the present) and time (the future). In this study, we did not balance the two time conditions. The present condition was composed of binary truth statements consisting of two possible options related to current world knowledge. The future condition consisted of two options that were not yet determined at the time of viewing, but which depended on the outcome of future mixed martial arts fights. According to the associative remote viewing (ARV) method, the binary outcomes of the present and future options were each associated with a photo, which had to be described by the viewers. An independent judge analyzed the viewers' qualitative reports through binary correspondence ratings amounting to a hit (1) or no hit (o) per trial. Independently of the time condition, a Psi effect could be observed. The hit rates of the judge ( 0.88 and 0.62 for the present and future, respectively) were significantly higher than the expected value (0.5) under the null hypothesis (present: $p<.001, \mathrm{ES}_{\mathrm{p}}=.73$; future: $p=.027, \mathrm{ESF}=.22$; binomial distribution). In addition, the hit rates in the two time conditions differed significantly from each other $\left(X^{2}=9.01 ; d f=1, p<0.003\right)$. The results confirm the hypothesis that Psi is not completely independent of the time dimension and that the hit rate is influenced by a priori target probabilities. With regard to the Informational Psi (IU) theory, we will discuss the implications of a probabilistic future for the understanding of Psi effects.

Keywords: Informational Psi; probabilistic future; associative remote viewing; anomalous cognition; collective unconscious
\end{abstract}




\section{INTRODUCTION}

\section{Theoretical Background}

In all time periods, humans have been fascinated by apparently inexplicable phenomena that lay outside of their ordinary understanding of everyday life. Temporal anomalies such as intuitions of spatially or temporally separated (i.e. future) events that become reality are extraordinary experiences (Barušs \& Mossbridge, 2016; Kripal, 2019; Radin, 2009; Wargo, 2018). Thanks to the development of academic experimental psychology in the late $19^{\text {th }}$ century, these anomalous or parapsychological experiences and effects have been studied academically (Cardeña, 2018; Mossbridge \& Radin, 2018; Roll, 1989). Jung (1963) used the term 'synchronicity' for those phenomena in which a connection of temporally separated events is experienced as personally meaningful, although there is no causal relationship between said events. In contrast, the classic 'transmission' model by J. B. Rhine postulates that causal information is conveyed by some yet-unknown carrier leading to extrasensory perception (for a discussion of causal and noncausal models, see Millar, 2019).

Mainstream science has always been critical or even dismissive of these purported experiences and positive research outcomes in parapsychology or, to use a more recent term, anomalous cognition (May \& Marwaha, 2014; Sommer, 2014). It has been argued that such experiences are due to distortions in subjective perception and memory and can find an explanation in the personality of the people making such extraordinary claims (Marks, 2000). Experimental results showing an anomalous effect are often categorically dismissed with statements such as Schwarzkopf's (2018): "No matter how strong the statistical evidence, if the hypothesis is impossible, it must necessarily be false." Alternatively, these results are dismissed because they stem from an allegedly inadequate application of statistical tests (Wagenmakers et al., 2011).

Regarding this latter criticism, the use of the "right" type of statistical test (e.g., Bayesian analysis vs. classical frequentist hypothesis testing) does not necessarily lead to a more straightforward picture. The setting of priors in Bayesian models is based on a selection pro- 
cess carried out by the researcher, which, in principle, should represent the state of scientific knowledge, but could also be interpreted as the quantification of a personal belief (Maier \& Dechamps, 2018; Tressoldi, 2011). Application of Bayesian statistics in meta-analyses has actually shown positive effect sizes for the anticipation of random future events (Bem et al., 2015), following initial significant results after classical hypotheses testing (Bem, 2011). Meta-analyses and systematic reviews of the existing literature reveal the presence of anomalous effects in many fields of Psi research (Cardeña, 2018; Mossbridge \& Radin, 2018).

Within the framework of the Star Gate program (May \& Marwaha, 2018), the systematic perception technique Remote Viewing (RV) has been developed, which allows for the investigation of a potentially anomalous information transfer. RV is defined as "mental faculty that allows a perceiver (a 'viewer') to describe or give details about a target that is inaccessible to normal senses due to distance, time, or shielding" (IRVA, 2020). From a psychological perspective, RV can be understood as anomalous cognition (May \& Marwaha, 2014), because humans become aware of something (a cognition) through a process that is so far unknown (anomalous). The concept of anomalous cognition and the general term Psi (Greek letter $\Psi$ ) are used synonymously for the same type of anomalous effects. Proof-oriented empirical studies show that RV-induced Psi effects can be detected (Marwaha \& May, 2019a). This means that, under specific conditions, the degree of correspondence between the perception of the subjects and a given target (e.g., an event, an outcome) is higher than expected by chance. Therefore, it has been suggested that future research should focus more on processoriented issues in order to gain more insights about the mechanisms behind this phenomenon (May \& Marwaha, 2015).

Over the years, scholars (Dunne \& Jahn, 2003; Krippner et al., 2019; Targ et al., 1995) conducted many different process-oriented as well as applied investigations using the remote or perceptual viewing technique, including distant intention approaches of one individual helping another while meditating (Schmidt et al., 2019). The fact that no convincing theory has been developed so far which could explain Psi effects within the framework of psychological knowledge is particularly noteworthy. Many models exist side by side (e.g., Carpenter, 2015; Walach et al., 2019), but there are no real decision criteria for comparative 
validity. An example of a current conceptualization that summarizes the empirical findings and attempts to explain Psi phenomena from a physical and neuroscientific perspective is the Informational Psi (IU) theory (Marwaha \& May, 2019a). ${ }^{1}$

The IU theory postulates that there is only one form of Psi while information builds the core of the Psi experience. It is the temporal localization of a target event in spacetime that defines whether we are dealing with perception of information in real-time (at present) or as precognition (from the future). There are a number of questions that remain unanswered within the $I \Psi$ theory (e.g., what is the source of the perceived information? Where does the information come from: actual or possible futures?). Therefore, the $I \Psi$ theory should not be seen as a final explanatory model, but rather as research in progress (Marwaha \& May, 2019b; see also the external comments on Marwaha \& May, 2019b, pp. 52-72).

The idea of the present study is to empirically test a time-related factor that has been discussed in a former study (Müller et al., 2019) as a limitation for RV—namely the probabilistic nature of the future, i.e. the fact that the future exists as various probabilities that can change in the course of time until an event actually happens. The opposite position is the assumption of a deterministic future in which everything is already predetermined or rather predestined. This distinction is relevant for the question of whether the RV-induced Psi effect is completely independent of time ( effect $_{\text {present }}=$ effect $_{\text {future }}$ ) or if there is a dependence on temporal characteristics (effect present $\neq$ effect $_{\text {future }}$ ). The possibility of predicting the future has been investigated in a series of studies (Harary \& Targ, 1985; Kolodziejzyk, 2012; Müller et al., 2019; Puthoff, 1984; Smith, 2009; Smith et al., 2014; Targ et al., 1995). In these studies, the observed hit rate, i.e. whether a prediction is correct or wrong, for binary events ${ }^{2}$ was significantly higher than the expected value under the null hypothesis.

The issue of whether it is generally possible to significantly predict a binary event in the future is only a secondary, albeit important aspect of this study because the empirical data are already indicative of a Psi effect. It is still unclear whether there are differences in hit rate between present and future viewing and whether this can be ascribed to the time factor (probabilistic future) or to other factors. If the probabilistic nature 
of the future contributes to the observed error variance, the hit rate for binary events in the present theoretically should be higher because additional aspects of the future have no influence on presently existing targets. Radin (1988) discovered that different a priori probabilities of targets in the future influenced the Psi quality in the present. To the best of our knowledge, no study yet has specifically investigated the hit rate difference for targets in the present and in the future. There are, however, meta-analyses on free-response and forced-choice studies (Storm et al., 2010, 2012) that generally found no significant differences in effect sizes (ES) between telepathy/clairvoyance (present targeting) and precognition (future targeting). Two options for possible study results are described below:

(1) Effect $_{\text {present }}=$ effect $_{\text {future }}$ : If the effects in both conditions were equal, $I \Psi$ would be time-independent because it would be similarly possible to correctly describe a target in the present and in the future. The error variance could be ascribed solely to factors that have no relation to time, such as mental noise as explained through cognitive processing of Psi information or the method for Psi induction. This result would support the determinist perspective according to which all future events are already predetermined and can be foreseen at any time with RV.

(2) Effect $_{\text {present }} \neq$ effect $_{\text {future }}$ : If the effects in both conditions were different, $I \Psi$ would be time-dependent because the condition (present vs. future) would influence how accurately a target can be described (provided that Psi effects are measured in both time conditions). We assume that the Psi effect is larger in the present condition than in the future condition (effect $t_{\text {present }}>$ effect $_{\text {future }}$ ). The difference between the Psi effects could give us a clue about how large the influence of the probabilistic future actually is. The result would falsify the assumption that $I \Psi$ is completely independent of the time dimension. In either case, the result would advance our understanding of $I \Psi$ and clarify the issue of whether the perceived information arises from actual or possible futures. Especially if we were to find Psi effects in both conditions and a significant difference between the two (effect present $>$ effect $_{\text {future }}$ ), we would conclude that Psi depends partially on the time dimension but is not absolutely constrained by it.

The overarching research questions for this study read as follows: 
(1) Is there a Psi effect using the remote viewing method for present and future targets; (2) Is there a difference between the Psi effects in the two time conditions, present and future? As operationalization, we use a study design to test whether a target in one of the two test conditions (present, future) has been described by a viewer. It has to be noted beforehand that we did not use a balanced design for the two conditions but for logistical reasons presented first the present and then the future condition. In case there are differences between conditions in outcome, we cannot make unambiguous statements about whether we have a time effect or an effect as a consequence of serial order, i.e. differences are potentially attributable to a learning or fatigue effect from condition 1 to 2 . In that way, this study design has the character of an exploratory (feasibility) study.

Moreover, the difficulty in the conception of such a study design is the fact that the outcomes of an RV session (descriptions and sketches) are qualitative data, which are not easy to analyze statistically in terms of target correspondence. This difficulty is rooted in the very nature of $1 \Psi$ and had already come up in early RV studies (Puthoff \& Targ, 1976; Schwartz, 1977). The impressions that a person generates during an RV session are mainly of a descriptive and sensory nature (e.g., descriptions of color, texture, or temperature) and contain in the majority of cases little or no analytical details about the target (e.g., mentions of names and functions). To ensure that a target has been described and that the descriptions contain more than just random correspondences, the data collection can be conceptualized in a so-called Associative Remote Viewing (ARV) design. A more detailed presentation of this design is provided in the Methods section of this paper. Importantly, we used subjects who had previous experience with the remote viewing technique.

The following hypotheses are tested in this study. The Psi hypothesis $\mathrm{H}_{1}$ is accepted, if an independent judge would be able to significantly identify one of two stimuli (binary rating) with the help of qualitative descriptions that subjects generated during a blind RV session in the course of $n=100$ trials. For $\mathrm{H}_{1}$ to be accepted, the observed hit rate of a judge should significantly differ from the expected value under the null hypothesis (no Psi, hit rate 0.5). The hypothesis is tested for all trials ( $n$ $=100$ ) and for both time conditions ( $n=50$ each). 
$\mathrm{H}_{1}$ : The observed hit rate of the judge is significantly higher than the expected hit rate of 0.5 (Psi effect).

To test the time hypothesis $\mathrm{H}_{2}$, the present and future hit rates (based on $n=50$ trials each) are compared. For $\mathrm{H}_{2}$ to be accepted, the hit rates in the two conditions should significantly differ from each other. From a theoretical perspective, it is assumed that effect $_{\text {present }}>$ effect $_{\text {future }}$.

H2: The hit rates in the two time conditions do significantly differ from each other in that the hit rate for the present effect is higher than the hit rate for the future effect (effect present $>$ effect $_{\text {future }}$ ).

\section{METHODS}

\section{Subjects}

In total, $n=5$ viewers took part in the study. They were selected on the basis of their training in the Coordinate Remote Viewing (CRV) protocol (Smith, 1986) and their practical experience with the method. The screening for suitable subjects took place through personal contacts within the German remote viewing community. The viewers had contact exclusively with the principal investigator (PI), who is the first author MM, and they did not know who the other participants were. The subjects were informed that their data would be used only to analyze the results of this study.

According to their own statements, all subjects took part in the study voluntarily and because of their personal motivation. For every trial the subjects received a compensation of $€_{7.50}$. The subjects who achieved the highest hit rates in each of the test conditions ('present' and 'future' condition) received an additional reward of $€_{150}$ each. The reward was meant to ensure that the subjects stayed motivated over the course of the study, especially over the last trials. The data collection took place in the period between October 2018 and March 2019.

\section{Targets and Stimuli}

Over the course of the study, the PI prepared in total $n=20$ targets (in essence, unfinished verbal statements, see below) with two options (two possible endings for each of the statements, see below) and, for 
each target/option pair, he selected two associated stimuli (in total $n$ $=40$ images). The targets (the statements) were related to the present (targets 1-10) and to the future (targets 11-20). The associated stimuli were digital images (photos) which had to be described by the viewers during data acquisition. Each of the $n=5$ subjects worked on all $n=$ 20 targets (in total $n=100$ individual trials). The following paragraphs provide a closer definition of the terms. In our prior ARV study (Müller et al., 2019), we had $n=50$ trials for detecting the target photos which were associated with the stock index going up or down. Since $n=50$ trials were sufficient to find significant effects in that study which employed the same dual ARV logic, we similarly chose $n=50$ trials for each of the two conditions ( 5 subjects $\times 10$ targets).

The targets in the present condition were open statements branching into two possible options, the correctness of which was already determined and unambiguously verifiable at the time of data acquisition (i.e., one option was clearly correct and one option was clearly incorrect). Example: "The current acting Federal President of Germany is ..." Option A: Frank Walter Steinmeier; Option B: Horst Seehofer. At the time of data acquisition, only option $A$ was correct, while option $B$ was clearly wrong. The targets in the future condition were statements branching into two options which were not yet determined at the time of data acquisition, but which became unambiguously verifiable after the future event had happened (i.e. one of the two options was clearly correct after the future event had taken place, while the other option was clearly wrong). Example: "The winner of the MMA ${ }^{3}$ fight on the 2nd February of 2019 is ..." Option A: Magomed Bibulatov; Option B: Rogério Bontorin. Both options were possible at the time of data acquisition. However, after the future event had happened, only one of the two options was correct. A draw is hardly ever an outcome in this type of fight, and this possibility did not occur for any of the ten targets of the future condition.

As associated stimuli, we used pairs of images that had to be as different as possible from each other (see Figure 1). The images were selected by the PI based on subjective criteria and on stimuli selection experience from prior studies (Müller \& Wittmann, 2017; Müller et al., 2019). In essence, the perceivable image contents of the two stimuli should be clearly distinguishable on various sensory dimensions 

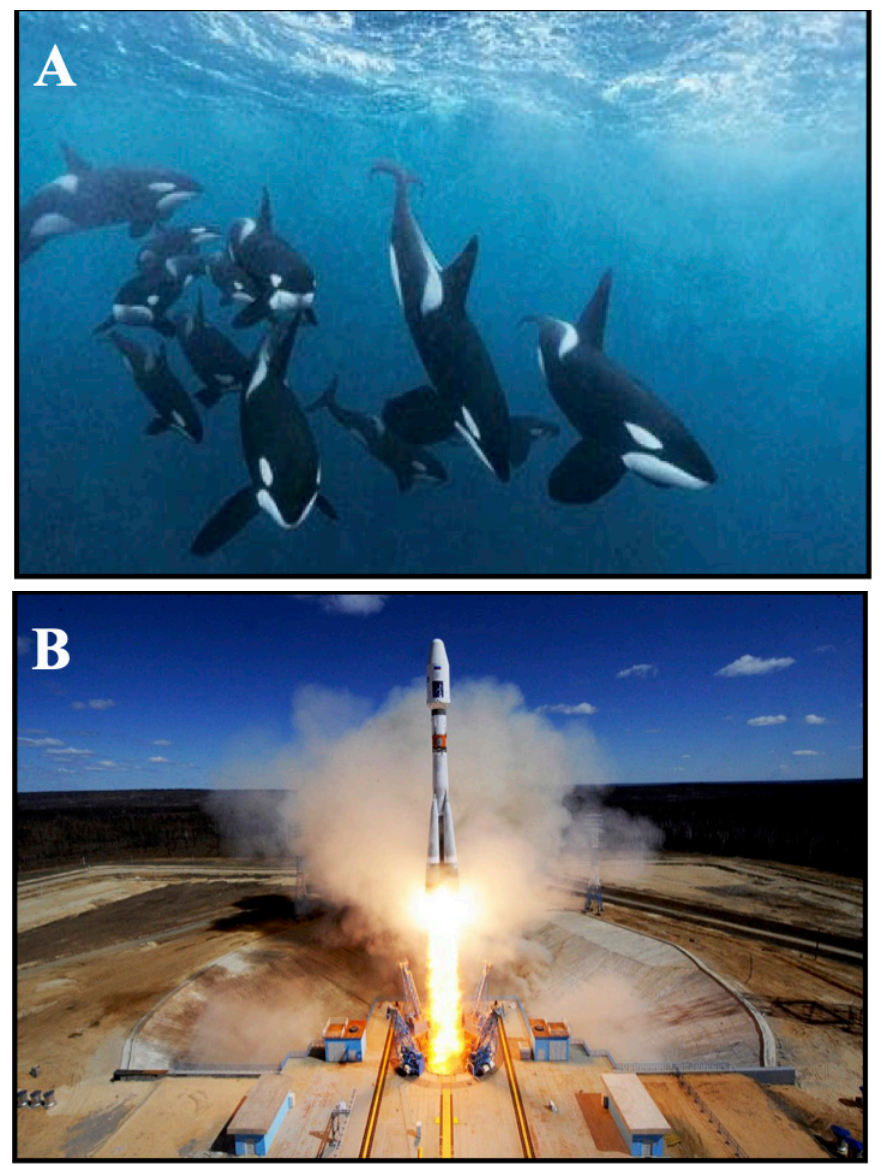

Figure 1. Example stimuli pair for a target. Image A: Orcas, Image B: Rocket launch. The stimuli differ from each other in various sensory aspects (e.g., color, surface quality, temperature).

(visual, auditory, tactile, kinesthetic, etc.). This is important to facilitate the subsequent analysis and to rule out the assumption that wrong decisions could depend on the stimuli being too similar. The stimulusstatement pairs were stored as electronic files (.pdf) on the computer of the $\mathrm{PI}$ and nobody else had access to them.

\section{Data Collection}

(1) Acquisition: For data acquisition, the subjects used the Coordinate Remote Viewing (CRV) protocol (Smith, 1986). The protocol 
consists of a systematic sequence of stages that a viewer has to work through during the RV session (circa 30 minutes). This method is thought to enhance the intuitive perception process of the viewer by focusing the attention on the target stimulus, categorizing the perceived impressions in (sensory) categories, and connecting the separate impressions into a coherent whole with the help of sketches and diagrams (May \& Marwaha, 2014). Moreover, the protocol contains mechanisms to bypass so-called analytical overlays (AOLs), i.e., rational inferences or fantasies. The aim of an RV session is to report information about the target stimulus in a way that is as unfiltered as possible (i.e., preferably without analytical overlays). The qualitative data in the form of descriptions and sketches (for example, see Figure 2) that result from

Figure 2. Example of qualitative Psidata. Descriptions and sketches a viewer generated during the RV session. This is an extract of a session which had been conducted on the target with the stimuli from Figure 1, Image B (rocket launch), which was associated with the correct target option.

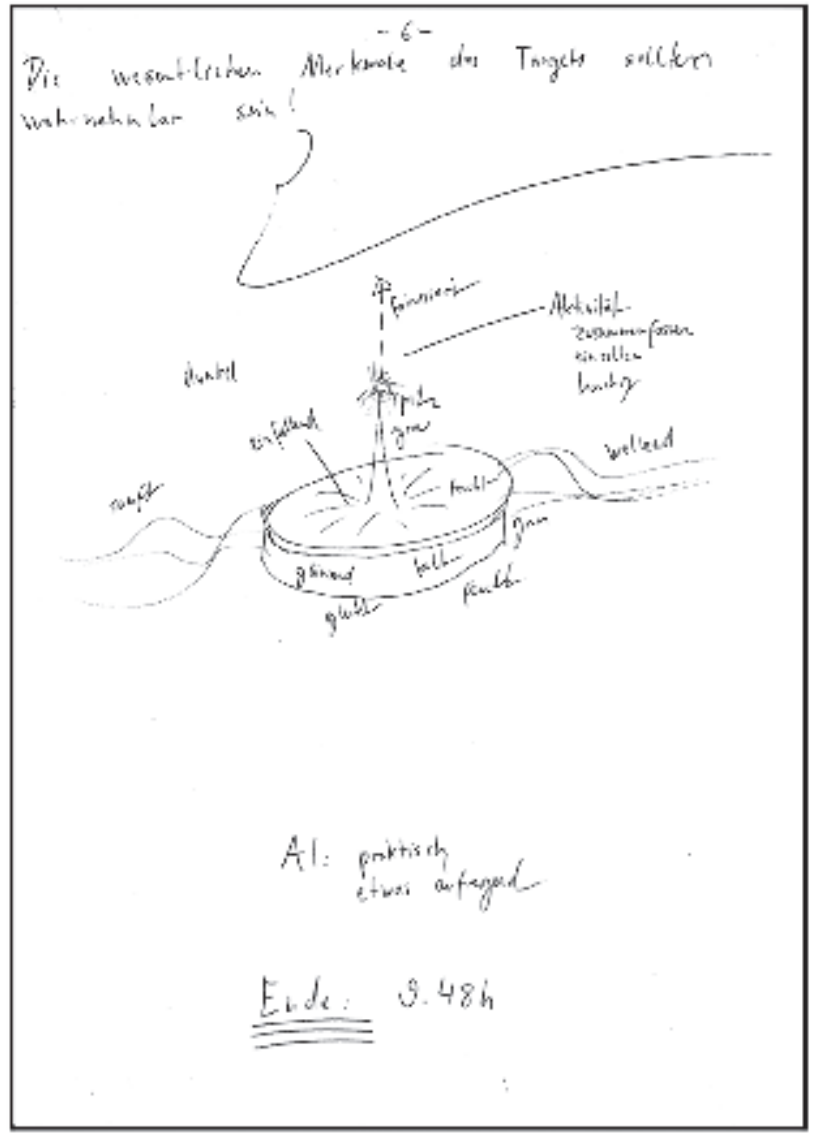


an RV session can be quantified with the help of a rating method for the statistical analysis. All participants had previous experience with the CRV protocol and used it consistently during the study.

(2) Rating: For the quantification of responses, we used a rating scale which made it possible to judge a session in terms of correspondence with the given stimuli (Figure 3). Our scale is based on the probed-and-tested scale by Targ et al. (1995, p. 374), which ranges from o to 7 points. Ours is a 5-point Likert scale with "No correspondence at all" (0) and "excellent correspondence" (5) as endpoints-with the option of marking half points (e.g., 2.5), for a total of 11 steps. Using this rating scale, the judge takes all descriptions and sketches of a session into account to estimate the overall correspondence of the information with the two images representing the two options. In the end, the judge has to make a forced-choice decision giving one of the two images at least half a point more than the other. The image with the higher point score is considered the target. From this judgment one can deduce whether a trial is a hit (1) or not (o).

0 - No correspondence (in essence no correct information)

1 - Low correspondence (relatively little correct and mainly wrong information)

2 - Correspondence (mix of correct and wrong information, mainly ambiguous)

3 - Correspondence (mix of correct and wrong information, mainly unambiguous)

4 - Good correspondence (mainly correct and relatively little wrong information)

5 - Excellent correspondence (in essence no incorrect information)

Figure 3. The rating scale used in this study. Shown here is the continuum $(0-5)$ the judge used for classification of the qualitative data. In brackets are the substantial definitions of the correspondence degrees.

We assumed any session to have at least a small correspondence value $(>0)$ with any image of the world because the perceivable world consists of a limited set of repeating impressions. Furthermore, we expected hardly any sessions to have an excellent correspondence (5) with an image because there are many psychological variables (e.g., mental noise or errors in the verbalization of impressions) which lead to 
distortions in perceptions and their descriptions. Therefore, the judge should have knowledge about the specific characteristics of qualitative Psi data to produce a useful rating. Eventually, the rating result is a numerical value that reflects the correspondence with the respective stimulus and can be used for statistical analyses.

(3) Evaluation: The difficulty in the acquisition and rating of Psi information lies in the descriptive-sensory or rather nonanalytical nature of the perceptions. To ensure that a Psi effect can be revealed, there has to be an evaluation design that allows for unambiguous decisions. With the Associative Remote Viewing (ARV) method, the viewer does not describe the target itself but instead one of two photos that are associated with the target. The association makes it possible to get information about the target by implication. For example, the target is the name of a specific person. This analytical information is hardly perceivable with RV. Therefore, one should formulate two options as follows: Option A being "the name of the person is X" and option B being "the name of the person is $Y$ ", where only one option is correct. Additionally, it is necessary to select two stimuli A and B (e.g., in the form of digital images) and associate them with the two options (note that the images have nothing to do with the person). In written form: If option A, then image A. If option B, then image B.

The viewer is instructed to describe only the image/stimulus that is associated with the correct option (blindly, i.e. without knowing the target or the images). The concrete task for the viewer (coded by a random target reference number) is: "Describe the image which is associated with the correct target option." Through the description of an image, the correct option is identified because it is determined that only one option is correct. The correct target option in the future condition is not yet determined at the time of the session but becomes clear after the event has happened in the future (i.e. one of the two fighters wins the MMA match). An advantage of this procedure is that, through the descriptions of the associated images, it is possible to make a clearer decision about the target. The simplest form is the conception of targets that have two options ( $A$ and $B$ ) because only two distinct images are needed.

Another advantage of the ARV design lies in the free target choice. The target can be related to the presence of something (e.g., the name 
of a person in a specific position, $A$ or $B$ ) or to an event in the future (e.g., the winner of a specific competition in the future, A or B). In both cases, it is important to make sure that the statements are binary. Furthermore, the target statements have to be unambiguously verifiable to allow an analysis of the data. When all other variables in both conditions are held constant (ultimately, photos have to be described) and a Psi effect is measured, a direct comparison between the two time conditions (present vs. future) becomes possible. A hypothetical difference would be solely attributable to the a priori probabilities of the targets that are defined by the time conditions.

\section{Experimental Procedure}

Over the course of the study, the experimental procedure was always the same and the functions of the $\mathrm{PI}(\mathrm{MM})$, the judge, and the five viewers, who all were experienced remote viewers, did not change. All seven individuals had at some point received a formal education in the remote viewing method. Regarding potential questionable research practices, it is important to mention that in this test design the PI functioned as a mediator between the viewers and the judge. Some of the viewers knew each other but were instructed not to exchange information regarding the study. Since knowledge of results was given only after the end of trial 10 (the present condition), communication would not have helped regarding better performance. Importantly, there was no communication between the viewers and the judge. During the course of the study, any information exchange concerning the study between the PI, the viewers, and the judge took place via email. In addition, researcher MW received the predictions about the prospective winner in advance of the fight (see below). The purpose of all these measures was to prevent unwanted information transfer. Thanks to these measures, the experiment fulfills accepted criteria for the testing of the Psi hypothesis (May \& Marwaha, 2015). Further details on control measures are described below.

Regarding the exact sequence of procedural steps:

(1) the PI selected one target and two stimuli (the photos), associated them randomly with the corresponding options ( $A$ and $B$; the statements), and generated a random target reference number. 
(2) The reference number, and only the reference number, was then submitted to the viewers via email. The email contained no additional information about the target or the two stimuli.

(3) With this number, the viewers conducted an RV session in their private surroundings (alone or with a partner) to collect information about the correct target stimulus. The viewers knew that the number was implicitly associated with the intention of the PI: "Describe the image which is associated with the correct target option." The viewers only knew that they were conducting an ARV session with two stimuli in the respective time condition, they did not know about the underlying statements or photos. With our blocked design, viewers were additionally informed before each block of trials that the ARV session was about present (trials 1-10) or future statements (trials 11-20).

(4) During the session, the viewer generated a transcript that he/ she sent back to the $\mathrm{PI}$ as a scanned document.

(5) The PI kept the transcripts for himself until one test series was completed in its entirety. One test series consisted of five sessions with the five viewers for a given target and a given option pair.

(6) As soon as one test series was conducted in full, the judge received the five transcripts of the viewers and the two images via email. The judge conducted a blind correspondence rating without knowing the target or the correct stimulus assignment. During the rating, the judge assessed the five transcripts and gave a correspondence rating on each of the given images ( $A$ and $B$ ) for each transcript. The judge was instructed to make sure that the rating between the images differed by at least 0.5 points so as to enable a binary analysis of the hit rate (hit/ no hit). Furthermore, the judge had to rate every transcript as neutrally as possible and without recourse to information from other transcripts.

(7) The ratings of the judge were sent back to the PI, who archived the values with the respective transcripts in a digital folder to which he alone had access.

In the present condition (targets 1-10), the viewers and the judge did not receive feedback about the correct stimulus after each trial. Only after completion of test series 10, i.e., after $n=50$ trials (for $n$ $=5$ viewers) were conducted, did the viewers receive comprehensive feedback with a personal analysis of their individual hit rate via email. The judge also received feedback for his rating after the completion of 
trial 10. This procedure was necessary to avoid possible information exchange between the subjects regarding the correct target images, as the trials were not conducted in parallel by all viewers.

In the future condition (targets 11-20), the viewers and the judge did receive feedback after each trial because the temporal characteristic of the dependent variable (i.e. the fact that it was in the future) eliminated the possibility of unwanted information transfer. The viewers were given one work week (from Monday to Friday) during the course of which they could conduct the sessions for the future target based on their own schedule. No matter what time they chose for the sessions, the prediction event was always in the future (either on Saturday or Sunday of the same week). After the event occurred (i.e. as soon as one fighter had won the MMA match), the result (hit/ no hit) could be verified, and feedback was given the following day. In the future condition, the feedback was given after each trial to keep the viewers motivated over the course of the study. The feedback could have no influence on the hit rate because all sessions of a test series and all ratings had already been conducted when the respective fight took place. As an additional control measure for the future condition, the predictions about the prospective winner of a fight were sent to a third party (author MW). MW was given the task of keeping track of all predictions and of the actual results over the course of the entire study. The following section provides a short explanation of the statistical analyses that were conducted after all results of the rating by the judge were transmitted to the PI.

\section{Data Analysis}

The Psi hypothesis $\mathrm{H}_{1}$ is tested against the expected value under the null hypothesis, which is a hit rate of 0.5 for the binary rating of $n$ $=100$ trials. There is a $50 \%$ probability that the judge assigns a higher correspondence to the correct target stimulus by chance only. The observed number of hits for exactly $n=100$ ratings should be significantly $k>50$ (binomial distribution) for $\mathrm{H}_{1}$ to be accepted. The observed number of hits in the respective time conditions with $n=50$ trials each should be $k>25$ for $\mathrm{H}_{1}$ to be accepted. Potential differences in hit rate between the two time conditions $\left(\mathrm{H}_{2}\right)$ are tested with chi-square $\left(X^{2}\right)$ statistics. 


\section{RESULTS}

Based on the viewers' transcripts, in 75 out of 100 trials the judge rated the correct target stimulus, which was associated with the actual target option, with the higher correspondence rating. This results in a hit rate of 0.75 , which is significantly higher than the expected value under the null hypothesis ( $p=1.9 \times 10^{-7}$; binomial distribution, $n=100$, $k=75, p=.5$ ). Across the individual trials, the five viewers show different hit rates: $V_{1}: 0.8 ; V_{2}: 0.9 ; V_{3}: 0.55 ; V_{4}: 0.7 ; V_{5}$ : 0.8 (see Table 1). An analysis of the individual viewers with binomial tests shows that Psi effects occurred independently for 4 out of 5 viewers. Descriptively, across all ratings, the mean correspondence rating of the session data is 2.81 for the actual target image and 1.88 for the nontarget (wrong) image.

TABLE 1

Hit Rates Sorted by Viewers

\begin{tabular}{ccccc}
\hline \hline Viewer & HR & Z & ES & p-Value \\
\hline 1 & 0.8 & 2.46 & 0.55 & $4.6 \times 10^{-3} \star \star$ \\
2 & 0.9 & 3.34 & 0.75 & $1.9 \times 10^{-4} \star \star \star$ \\
3 & 0.55 & 0.22 & 0.05 & $1.6 \times 10^{-1}$ \\
4 & 0.7 & 1.57 & 0.35 & $3.7 \times 10^{-2} \star$ \\
5 & 0.8 & 2.46 & 0.55 & $4.6 \times 10^{-3} \star \star$ \\
\hline
\end{tabular}

Note: Observed hit rates (HR), z-values (z), Effect Sizes (ES), and $p$-values sorted by viewers.

Analyses for each viewer are based on $n=20$ measurements.

${ }^{\star} p<.05,{ }^{* *} p<.01,{ }^{* \star *} p<.001$ point to significant results and therefore a psi effect.

For the present condition, in 44 out of 50 trials the judge rated the correct target stimulus, which was associated with the actual target option, with the higher correspondence rating (see Table 2). This results in a hit rate of 0.88 , which is significantly higher than the expected value under the null hypothesis $(p<.001$; binomial distribution, $n=$ 50, $k=44, p=.5)$. The effect size of $\mathrm{ES}_{\mathrm{p}}(d)=0.73$ corresponds to a medium effect (Cohen, 1988). For the future condition, in 31 out of 50 
trials the judge rated the correct target stimulus, which was associated with the actual target option, with the higher correspondence rating (i.e., 31 out of 50 predictions of future events were correct). This results in a hit rate of 0.62 , which is significantly higher than the expected value under the null hypothesis $(p=.027$; binomial distribution, $n=$ $50, k=31, p=.5)$. The effect size of $\mathrm{ES}_{\mathrm{F}}(d)=.22$ corresponds to a small effect. Consequently, the hypothesis $\mathrm{H}_{1}$ can be accepted (for both time conditions). In the present condition as well as in the future condition, Psi effects could be observed.

A two-way chi-square test for the factor time (present, future) and the hit rate $(1,0)$ shows a significant hit rate difference $\left(X^{2}=9.01 ; d f=\right.$ $1, p<0.003)$ between the two time conditions. Descriptively, the mean difference between the ratings for the target image and the rating for the wrong image is higher for the present condition (3.12 vs. 1.59) than for the future condition (2.5 vs. 2.16).

\section{DISCUSSION}

The goal of this study was to investigate whether and to what extent Psi effects assessed with the remote viewing technique depend on the time dimension. In other words, we wanted to investigate whether the hit rate differed for present and future conditions. The results show that there is an overall Psi effect across both time conditions and that the hit rates between the two time conditions were significantly different. Therefore, hypotheses $\mathrm{H}_{1}$ and $\mathrm{H}_{2}$ both could be accepted. The following sections discuss possible interpretations and consequences of these results in the context of the $I \Psi$ theory (Marwaha \& May, 2019a). Before that, we will discuss possible limitations of the experimental design and consider whether it was appropriate for answering the research question.

Because we selected experienced remote viewers for the study, the subjects had the necessary competence to conduct the sessions on their own. Furthermore, through the description of the images it was possible to make clear statements (hit/no hit) regarding the associated targets for both time conditions. The analysis with the rating scale and the use of an independent judge, who was an experienced remote viewer himself, satisfied all requirements for an appropriate use 
of the information derived from the qualitative data. Thanks to the experimental design (randomization of stimuli, blind conditions during acquisition and analysis, etc.), the internal validity of the data can be assumed because potential interference factors (e.g., information leak) were controlled for. This means that if an effect is observed, it is reasonable to assume that it is due to a Psi process.

The serial order of the targets for the present condition (targets 1-10) and for the future condition (targets 11-20) leaves room for some criticism. We could have implemented a balanced design where the sequence of the present and future conditions would have been controlled for, but we did not. The rationale behind this decision was our desire to clearly separate the two time conditions for the three parties involved: the PI, the five viewers, and the judge. A potential order effect is relevant for the interpretation of the time effect. The viewers might have been more enthusiastic and motivated for the first batch of trials (present condition, targets 1-10) than for the second batch (future condition, targets 11-20). The viewers might have worked with less concentration in the later trials which in turn might have caused a reduction of the Psi quality and the lower hit rate for the future condition.

With the monetary incentive of $€_{7.50}$ for every completed trial and an additional reward $\left(\epsilon_{150}\right)$ for the highest hit rates in each of the two time conditions, we wanted to make sure that the participants were committed to performing to the best of their abilities. The viewers did not receive any feedback about the hit rates of the other participants during data acquisition. Therefore, the motivation to win the rewards should have stayed consistent for each viewer over time. The positive attitude of the viewers becomes evident from the transcripts of the $\mathrm{RV}$ sessions. This is evident through inspection of the qualitative data which has detailed descriptions and sketches from start to finish. Objectively, the judge rated the correspondence of the transcripts with the images (independent of whether it was a target stimulus or not) in both time conditions as nearly identical ( 2.36 vs. 2.33 for the present and future conditions, respectively). This means that, based on the ratings provided by the judge, the perceptions of the viewers in the present condition had the same level of quality as those in the future condition.

Nevertheless, a potential order effect through the target sequence cannot be completely excluded. This should be taken into consideration 
when interpreting the time effect. A replication study implementing a counterbalancing design is needed to fully test the hypothesis of time differences. A further difference between the time conditions is related to the time of feedback. In the present condition, comprehensive feedback with a personal analysis of the individual hit rate was given via email only after all trials (1-10) had been completed. For the future condition, the viewers and the judge did receive feedback after each trial (11-20). This more immediate feedback of performance for the future condition could have influenced remote viewing behavior differently, i.e. trial-to-trial adjustments, from the overall feedback given only at the end for the present condition. This leaves room for a stricter methodological isomorphism in a replication study.

The issue of replicability in Psi research is of the highest importance. Our results are meaningful only if future attempts replicate the outcome of this study with redesigned and preregistered replication studies (Marks, 2020). Additionally, it could be useful to implement a design in which the viewer is not aware of the time condition of the trial. This would ensure that the observed effects are solely attributable to the manipulation of the independent variable "time." It is still interesting to note how the qualitative data have nearly identical rating values for both time conditions, which speaks against a mere order effect. The fact that the viewers generated stimuli descriptions that had equal quality in both time conditions, but significantly different hit rates, could be an indication that the outcome of the event in the future was not completely predictable at the time of the session. Therefore, the time factor could have had an influence on the size of the Psi effect in the time conditions.

\section{Considerations on the Psi Effect}

The Psi hypothesis $\mathrm{H}$ could be accepted overall and for both time conditions because the hit rates of the judge were significantly higher than the expected value under the null hypothesis for binary ratings. Working blindly (i.e., without knowing the correct stimulus), the judge rated the correspondence for the correct image significantly more often than for the wrong image. These effects are consistent with findings of other studies (e.g., Dunne \& Jahn, 2003; May \& Marwaha, 
2014) showing that under specific conditions subjects are able to receive information from spatially and temporally separated targets. The observed Psi effects in this study lie in the small-to-medium size range, which is what was expected given the choice of trained subjects (Utts, 1996).

We will now tentatively discuss the question of how the viewers were able to perceive the stimuli and, furthermore, which source of information they potentially used to correctly identify a target significantly often. Because the viewers did not perceive a target directly but with the help of associated images, a connection between targets, stimuli, and the viewers has to be assumed. Because of the study design, the viewers were blind toward both the targets and the stimuli during data acquisition. The perceptions described must have come into the information processing system of the viewers by other means, enabling them to generate significantly correct descriptions of the stimuli, which in turn were associated with the correct target options. The $I \Psi$ theory postulates a physical information transfer of which the characteristics are unknown and that the source of the Psi information can be localized as a "distant point in spacetime" (Marwaha \& May, 2019b, p. 15).

From a psychological perspective, the qualitative data (perceptions of the viewers) provide starting points for the identification of a potential Psi information source. This type of perception is not entirely unlike sensory perception; in fact, there are some parallels. The impressions a viewer generates during an RV session are mainly of a descriptive-sensory, or nonanalytical, nature. The viewer reports what they intuitively perceives when their attention is directed on the target (bottom-up processing) and preferably without interpreting concrete content into the perceptions (top-down processing). Practice shows that the exclusion of any analytical processes (e.g., memory, logical reasoning, etc.) is possible only to a given extent. The analytical processes that cannot be prevented are known as mental noise-an error source for RV. The acquisition of target information can be compared with tapping into a signal that, in addition to other cognitive processes, should be perceived as much as possible without filters (i.e., without analytical overlays). Marwaha and May (2019b) assume that $I \Psi$ is, just like the other human senses, a signal-based sensory system with a clear 
source as well as transmission and detection mechanisms. According to Carpenter (2015), Psi processes are inherently, but unconsciously, involved as the "leading edge" in any conscious perceptual and decision process.

The signal should arise from a distant point in spacetime but the perceptions of the viewers are always local (Marwaha \& May, 2019a, 2019b). This assumption presupposes an external information transfer between target and viewer that has not been shown yet. Alternatively, one could consider the source of the Psi signal not as distant but, just like the perception of Psi information, as a nonlocal process. 'Nonlocal' means that the Psi information is already present for the viewer and is brought to his awareness during the RV session. In contrast to the $I \Psi$ theory, this explanatory model presupposes an internal information access, whereas the possibility of an external information transfer in the form of an external signal is rejected. Furthermore, this assumption presupposes the existence of a part of the psyche that contains information beyond the mental limitations of the individual which is inaccessible by means of conscious processes. Inspired by the conceptual analyses of C. G. Jung (1959, 1963), we could think of the unconscious mind and, additionally, a so-called "collective unconscious," as the source of Psi information. By collective unconscious, Jung meant an omnipresent construct that is an unconscious part of the psychic system of every person, but which has a nonpersonal or rather collective character. During the RV session, the viewer might have access to this unconscious construct to receive or become aware of information about a target. Importantly, this idea is not based on the assumption of a local-causal signal transfer from spatial or temporally remote targets but instead on the inherent nonlocality of psychic phenomena which is independent of material causes (Walach, 2020).

Although the theories presented are coherent, they can hardly be considered congruent with the world, i.e., that elements of the theory show an isomorphism with empirically shared and universally accepted evidence of underlying structures and processes. The explanatory model suggested above is mainly based on observations of the phenomenal characteristics and of the analysis of qualitative Psi data. A comprehensive explanation of Psi in relation to the concept of the collective unconscious is beyond the scope of this empirical study. 
Nevertheless, the possibility of an internal signal in addition to the assumption of an external signal (as postulated in the $I \Psi$ theory) should be considered. In addition to the physical and neuroscientific domains, psychology or analytical psychology also should eventually provide an explanation for $I \Psi$ effects.

\section{Considerations on the Time Effect}

The time hypothesis $\mathrm{H}_{2}$ was accepted because the hit rates in the present and future conditions differed significantly from each other. The Psi effect in the present condition $\left(\operatorname{ES}_{p}(d)=0.73\right)$ showed a higher effect size than the Psi effect in the future condition $\left(\operatorname{ES}_{F}(d)=0.22\right)$. The Chi-square statistics for the percentage of hits between the time conditions provides evidence for significant differences. These findings support the theoretical assumption that RV is not completely independent of the time dimension. There is ample evidence for remote viewing of future targets in prior ARV studies (e.g., Müller et al., 2019; Smith et al., 2014; Targ et al., 1995). The effect size in our study $(E S(d)=0.22)$ was indeed relatively small compared with prior studies. The probabilistic nature of the future would explain the difference between the time conditions in our study. Based on the data, it is also possible to discuss whether the Psi effect is dependent or independent of a priori target probabilities. According to Targ and Targ (1986), Psi effects are independent of a priori defined target probabilities, whereas Radin (1988) observed opposite results.

Bearing the methodological caveats in mind, as discussed above, the fact that the variables pertaining to the identification of images were kept constant in both time conditions, the difference could potentially be attributed to the time factor. The future condition negatively influenced the hit rate, i.e., the viewers described the stimulus associated with the correct target option in the future less frequently. The time of the information acquisition can be considered crucial: In the present condition, the correct target option is already determined during the RV session. In the future condition, the correct target option is not yet determined during the RV session, i.e., the target event is open for possible changes until it finally happens. At the time of the RV session about a target in the future, the viewer does not describe the defi- 
nite target option as he/she does in the present condition, but merely the most probable target option. This leads to the conclusion that the future exists in probabilities and is not completely determined. A possible explanation for the failure to detect a strong future effect could be the nature of the prediction objects. In contrast to many previous ARV studies, which used the future course of a stock index (up, down) as the target (e.g., Müller et al., 2019; Smith et al., 2014; Targ et al., 1995), we used martial arts fights and their future winners as targets in this study. Based on the concept of the probabilistic future, it is obvious that different prediction objects underlie different a priori probabilities for the target events. We are aware that this interpretation is speculative, but it may be a helpful framework for the design of studies which attempt to vary future conditions with more or less determined futures. Potentially, there could be an expectation effect as precognition (future condition) could be perceived to be more difficult than real-time (present condition) psi (Storm \& Thalbourne, 2003). One solution would be to hide the type of condition (present or future) from the viewers, but it could be argued that a viewer could still sense the difference. The influence of the probabilistic future on Psi effects can be investigated for time-related variables because it is reasonable to assume that there is a predictable but "open future." For some targets, the hit rate is equal to random guessing because volatile events are not significantly foreseeable whereas other events are. From our theoretical deliberations, it seems plausible that the perception of the viewer and the relating hit rate of the judge are influenced by the predictability of a target in the future-depending on whether the a priori probabilities change after data acquisition (a posteriori) or not. This issue can be investigated in follow-up studies with the use of experimentally manipulated a priori target probabilities. This is consistent with the previous study by Radin (1988), who also assumed that the Psi effect depends on a priori probabilities of a future target.

The results described here have consequences for the controversially discussed topic of Psi and time (Barušs \& Mossbridge, 2016). The controversy can be summarized as "from where does the information arise-from an event or from later feedback, from actual or possible futures?" (Marwaha \& May, 2019a, p. 40). In various studies, no connection between Psi quality and feedback could be found (May et al., 
2014; Müller et al., 2019; Targ et al., 1985). In the present study, we gave feedback for both time conditions, but the hit rates still significantly differed. Therefore, feedback cannot be used as an axiomatic explanation for observed Psi effects. In this context, and under the assumption of the probabilistic future, a hypothetical "future answer book" (Marwaha \& May, 2019b, p. 104) cannot be the principal source of the Psi information. In sum, the data indicate that the perceived Psi information describes nothing but a probable future. As discussed above, our data results have an exploratory character and replication studies need to follow. It remains unclear whether the viewer perceives the information from the target event itself (or rather the associated stimuli) or from another source.

\section{CONCLUSION}

With a systematic application of the CRV protocol, this study was able to confirm that humans can gather information that would not be accessible to them through their ordinary "five senses" or their analytical mind. Potentially, we tapped into an anomalous type of cognition that apparently functions independently of space and time. The results regarding the time effect show that $I \Psi$ is not independent of the time dimension and that the future most likely consists of probabilities. Marwaha and May (2019b, p. 107) write: "The crux of the psi experience is indeed understanding the nature of time and information." Therefore, future studies could experimentally manipulate the a priori target probabilities and measure their effect on the qualitative Psi data. To finally achieve a holistic understanding of $I \Psi$, the source of the Psi information (internal vs. external) has to be identified. Only with a theory that can convincingly explain the source of the Psi information in connection with accepted knowledge about the world, could the controversial research subject of Psi and its observed effects be accepted by the scientific community (Mossbridge \& Radin, 2018; Schwarzkopf, 2018).

\section{NOTES}

1 Informational psi $(I \Psi)$ is defined as the transfer of information, which is based on entropic considerations, arising from a distant 
point in spacetime leading to the local acquisition of noninferential information by an atypical perceptual ability (Marwaha \& May, 2019a, p. 16).

2 According to the null hypothesis (no Psi effect), the expected hit rate for binary events is 0.5 , which means that $50 \%$ of all predictions are correct by chance.

$3 \mathrm{MMA}=$ Mixed Martial Arts is a full-contact sport similar to boxing, but which also includes elements of other combat sports (among which are ground fighting). Victory is normally achieved through a knockout (KO), or one fighter admits defeat, or based on points.

\section{REFERENCES}

Barušs, I., \& Mossbridge, J. (2016). Transcendent mind: Rethinking the science of consciousness. American Psychological Association.

Bem, D. J. (2011). Feeling the future: Experimental evidence for anomalous retroactive influences on cognition and affect. Journal of Personality and Social Psychology, 100(3), 407-425. https://doi.org/10.1037/a0021524

Bem, D., Tressoldi, P. E., Rabeyron, T., \& Duggan, M. (2015). Feeling the future: A meta-analysis of 90 experiments on the anomalous anticipation of random future events. F1000 Research, 4(1188). https://doi.org/10.12688/ fioooresearch.7177.2

Cardeña, E. (2018). The experimental evidence for parapsychological phenomena: A review. American Psychologist, 73(5), 663-677. https://doi.org/10.1037/ampooo0236

Carpenter, J. C. (2015). First sight: ESP and parapsychology in everyday life. Rowman \& Littlefield.

Cohen, J. (1988). Statistical power analysis for the behavioral sciences (2nd ed.). Erlbaum.

Dunne, B. J., \&Jahn, R. G. (2003). Information and uncertainty in remote perception research. Journal of Scientific Exploration, 17(2), 207-241.

Harary, K., \& Targ, R. (1985). A new approach to forecasting commodity futures. Psi Research, 4, 79-85.

International Remote Viewing Association (IRVA). (2020). What is remote viewing? http://www.irva.org/remote-viewing/definition.html

Jung, C. G. (1959). The concept of the collective unconscious. Collected Works (pp. 99-104; Vol. 9.I.). http://www.bahaistudies.net/asma/The-Concept-of-theCollective-Unconscious.pdf

Jung, C. G. (1963). Memories, dreams, reflections. Pantheon.

Kolodziejzyk, G. (2012). Greg Kolodziejzyk's 13-year associative remote viewing experiment results. The Journal of Parapsychology, 76(2), 349-368.

Kripal, J. J. (2019). The flip: Epiphanies of mind and the future of knowledge. Bellevue Literary Press. 
Krippner, S., Saunders, D. T., Morgan, A., \& Quan, A. (2019). Remote viewing of concealed target pictures under light and dark conditions. EXPLORE, 15(1), 27-37. https://doi.org/10.1016/j.explore.2018.07.001

Maier, M. A., \& Dechamps, M. C. (2018). Observer effects on quantum randomness: Testing micro-psychokinetic effects of smokers on addiction-related stimuli. Journal of Scientific Exploration, 32(2), 265-297.

Marks, D. F. (2000). The psychology of the psychic (2nd ed.). Prometheus Books.

Marks, D. F. (2020). Psychology and the paranormal: Exploring anomalous experience. Sage.

Marwaha, S. B., \& May, E. C. (2019a). Informational Psi: Collapsing the problem space of Psi phenomena. Zeitschrift für Anomalistik, 19, 12-51. https://www. anomalistik.de/images/pdf/zfa/zfa2019_12_o12_marwaha_may.pdf

Marwaha, S. B., \& May, E. C. (2019b). Author's Response: Signals: A mechanism to understand Psi phenomena. Zeitschrift für Anomalistik, 19(1\&2), 73-112. DOI: 10.23793/zfa.2019.73

May, E. C., \& Marwaha, S. B. (Eds.). (2014). Anomalous cognition: Remote viewing research and theory. McFarland.

May, E. C., \& Marwaha, S. B. (2015). Next step: Process-oriented research: Guidelines for experimenters. In E. C. May, \& S. B. Marwaha (Eds.), Extrasensory perception: Support, skepticism, and science: Vol. 2 Theories of psi (pp. 329-354). Praeger Publications.

May, E. C., \& Marwaha, S. B. (Eds.). (2018). The Star Gate archives: Reports of the United States government sponsored psi program, 1972-1995. Vol. 1: Remote viewing, 1972-1984. McFarland.

May, E. C., Lantz, N. D., \& Piantanida, T. (2014). Feedback considerations in anomalous cognition experiments. In E. C. May, \& S. B. Marwaha (Eds.), Anomalous cognition: Remote viewing research and theory (pp. 104-116). McFarland.

Millar, B. (2019). Observational theories of Psi. In Psi encyclopedia. The Society for Psychical Research, London. https://psi-encyclopedia.spr.ac.uk/articles/ observational-theories-psi

Mossbridge, J. A., \& Radin, D. (2018). Precognition as a form of prospection: A review of the evidence. Psychology of consciousness: Theory, research, and practice, 5(1), 78-93. https://doi.org/10.1037/cnsoooo121

Müller, M., \& Wittmann, M. (2017). Remote viewing: Eine proof-of-principlestudie. Zeitschrift für Anomalistik, 17, 83-104. https://www.anomalistik.de/ images/pdf/zfa/zfa2017_12_o83_mueller_wittmann.pdf

Müller, M., Müller, L., \& Wittmann, M. (2019). Predicting the stock market: An associative remote viewing study. Zeitschrift für Anomalistik, 19(3), 326-346. https://doi.org/10.23793/zfa.2019.326

Puthoff, H. E. (1984). ARV applications. In W. G. Roll (Ed.), Research in parapsychology (pp. 121-122). Scarecrow Press.

Puthoff, H. E., \& Targ, R. (1976). A perceptual channel for information transfer over 
kilometer distances: Historical perspective and recent research. Proceedings IEEE, 64(3), 329-354. https://doi.org.10.23793/zfa.2019.73

Radin, D. (1988). Effects of a priori probability on psi perception. Journal of Parapsychology, 52, 187-212.

Radin, D. (2009). The conscious universe: The scientific truth of psychic phenomena. HarperOne.

Roll, W. G. (1989). This world or that. An examination of parapsychological findings suggestive of the survival of human personality after death [dissertation]. Lund University.

Schmidt, S., Jo, H.-G., Wittmann, M., Ambach, W., \& Kübel, S. (2019). Remote meditation support-A multimodal distant intention experiment. EXPLORE, 15(5), 334-339. https://doi.org/10.1016/j.explore.2018.12.002

Schwartz, S. A. (1977). Two application-oriented experiments employing a sub-marine involving a novel remote viewing protocol, one testing the ELF hypothesis. Invited paper for The Philosophical Research Society Conference on Extraordinary Human Functioning. https://doi.org/10.13140/ RG.2.2.22217.95846

Schwarzkopf, D. S. (2018). On the plausibility of scientific hypotheses: Commentary on Mossbridge and Radin (2018). Psychology of Consciousness: Theory, Research, and Practice, 5(1), 94-97. https://doi.org/10.1037/cnso000125

Smith, C. C., Laham, D., \& Moddel, G. (2014). Stock market prediction using associative remote viewing by inexperienced remote viewers. Journal of Scientific Exploration, 28(1), 7-16.

Smith, P. H. (1986). Coordinate ("controlled") remote viewing manual. Defense Intelligence Agency. https://rviewer.com/controlled-remote-viewing-manualbackground-and-overview/coordinate-controlled-remote-viewingmanual-table-of-contents/

Smith, P. H. (2009). Is physicalism "really" true? An empirical argument against the universal construal of physicalism. The University of Texas at Austin.

Sommer, A. (2014). Psychical research in the history and philosophy of science. An introduction and review. Studies in History and Philosophy of Biological and Biomedical Sciences, 48, 38-45. https://doi.org/10.1016/j.shpsc.2014.08.004

Storm, L., \& Thalbourne, M. A. (2003). Perceived complexity, perceived task difficulty, and other states of mind: The influence of mental states on psi outcomes. Journal of the American Society for Psychical Research, 97, 155-174.

Storm, L., Tressoldi, P. E., \& Di Risio, L. (2010). Meta-analysis of free-response studies 1992-2008: Assessing the noise reduction model in parapsychology. Psychological Bulletin, 136(4), 471-485. https://doi:10.1037/aoo19457

Storm, L., Tressoldi, P. E., \& Di Risio, L. (2012). Meta-analyses of ESP studies, 19872010: Assessing the success of the forced-choice design in parapsychology. Journal of Parapsychology, 76, 243-273.

Targ, E., \& Targ, R. (1986). Accuracy of paranormal perception as a function of 
varying target probabilities. Journal of Parapsychology, 50(1), 17-28.

Targ E., Targ R., \& Lichtarge, O. (1985). Realtime clairvoyance: A study of remote viewing without feedback. Journal of the Society for Psychical Research, 79, 493-500.

Targ, R., Katra, J., Brown, D., \& Wiegand, W. (1995). Viewing the future: A pilot study with an error-detecting protocol. Journal of Scientific Exploration, 9(3), 367-380.

https://www.scientificexploration.org/docs/9/jse_o9_3_targ.pdf

Tressoldi, P. E. (2011). Extraordinary claims require extraordinary evidences: The case of non-local perception, a classical and Bayesian review of evidence. Frontiers in Psychology, 2(117). https://doi.org/10.3389/fpsyg.2011.00117

Utts, J. (1996). An assessment of the evidence for psychic functioning. Journal of Scientific Exploration, 10, 3-30. https://www.scientificexploration.org/ docs/10/jse_10_1_utts.pdf

Wagenmakers, E.-J., Wetzels, R., Borsboom, D., \& van der Maas, H. L. J. (2011). Why psychologists must change the way they analyze their data: The case of psi: Comment on Bem (2011). Journal of Personality and Social Psychology, 100(3), 426-432. https://doi.org/10.1037/aoo22790

Walach, H. (2020). Inner experience-Direct access to reality: A complementarist ontology and dual aspect monism support a broader epistemology. Frontiers in Psychology, 11(640). https://doi.org/10.3389/fpsyg.2020.00640

Walach, H., Horan, M., Hinterberger, T., \& von Lucadou, W. (2020). Evidence for anomalistic correlations between human behavior and a random event generator: Result of an independent replication of a micro-PK experiment. Psychology of consciousness: Theory, research, and practice, $7(2), 173-188$. https://doi.org/10.1037/cnsoooo199

Wargo, E. (2018). Time loops: Precognition, retrocausation, and the unconscious. Anomalist Books. 